\title{
EL USHNU Y LA ASTRONOMÍA DE HORIZONTE EN VIÑA DEL CERRO
}

\author{
THE USHNU AND HORIZON ASTRONOMY IN VIÑA DEL CERRO
}

\author{
Ricardo Moyano ${ }^{1}$
}

\begin{abstract}
En este trabajo se presentan los resultados obtenidos a partir del análisis de las orientaciones de la arquitectura reconstruida en el centro metalúrgico Diaguita-Inka Viña del Cerro, sector medio de la cuenca del río Copiapó, III Región de Atacama, norte de Chile. Estos resultados sugieren que la plataforma que formaba parte del ushnu, y los vanos de acceso a la kancha y recintos de la unidad A, pudieran estar relacionados con la elaboración y ajuste de un calendario solar de horizonte y con determinadas montañas del paisaje local. El ushnu en Viña del Cerro, además de cumplir con funciones políticas y administrativas establecidas por el Tawantinsuyu en el lugar, habría servido como un axis mundi, escenario para las principales ceremonias religiosas vinculadas con la observación de los solsticios y el culto a las huacas de los cerros Calquis y Potro.
\end{abstract}

Palabras claves: $u s h n u$, marcadores de horizonte, Viña del Cerro.

In this paper, I present the results obtained from an analysis of orientations of the reconstructed architecture in the Diaguita-Inka metallurgical center of Viña del Cerro located in the central valley of the Copiapó River, III Atacama Region in Northern Chile. These results suggest that the platform that formed part of the ushnu as well as the access doors of the kancha and structures of Unit A could be linked to the creation and adjustment of a solar horizon calendar and to specific mountains in the local landscape. The ushnu in Viña del Cerro, in addition to complying with political and administrative functions established by the Tawantinsuyu, could have served as an axis mundi, a place for principal religious ceremonies related to solstice observation and worship of the huacas of the mountains Calquis and Potro.

Key words: Ushnu, horizon landmarks, Viña del Cerro.

Viña del Cerro, también llamado Cabeza de Puerco o Apacheta, corresponde a un centro metalúrgico Diaguita-Inka (1.470-1.536 d.C.) ubicado en el sector medio de la cuenca del río Copiapó, III Región de Atacama (Figura 1). El sitio se localiza sobre un espolón rocoso a los pies del cerro Calquis, en cuyas cercanías se habría ubicado un pueblo de indios conocido históricamente como Painegue (Urízar 2002). A finales de la década de 1950 Jorge Iribarren describe lo que para él eran "construcciones indígenas" y realiza el primer levantamiento topográfico con ayuda del ingeniero Hans Niemeyer (Iribarren 1958). El mismo Niemeyer a finales de la década de 1960 inició las primeras excavaciones sistemáticas (Figura 2), luego entre 1979 y 1980 realizó un nuevo levantamiento topográfico del centro metalúrgico (Figura 3) (Niemeyer 1986). En 1982 bajo la supervisión del Museo Regional de Atacama, el Instituto de Investigaciones Arqueológicas y Restauración Monumental de la Universidad de Antofagasta llevó a cabo los trabajos de restauración arquitectónica, respetando la orientación original, tras lo cual el sitio adquiere las características que hoy conocemos (Niemeyer et al. 1983, 1984). Los trabajos realizados por Hans Niemeyer y colaboradores en Viña del Cerro (Niemeyer 1986; Niemeyer et al. 1993 [1991]) sugerían que la plataforma identificada como un ushnu habría simbolizado la autoridad del Inka cumpliendo funciones políticas, administrativas y religiosas vinculadas con el control de las materias primas, la contabilidad y la producción metalúrgica. Esta interpretación no tomó en cuenta dos cosas. Primero, el concepto de ushnu es más amplio que la sola plataforma e incluiría posiblemente un agujero y sistema de drenaje, además de un gnomon ${ }^{1}$ como señalan algunos trabajos para la ciudad del Cusco (Zuidema 1980, 1981, 1989, 2009). Segundo, no se tomó en cuenta la importancia de algunos elementos del entorno local (Bustamante 2004; Pino 2004, 2005), p.ej. cerros, formas de la topografía, el Sol, la Luna y posiblemente algunas zonas de la Vía Láctea, que en un sentido amplio tuvieron y tienen importancia

$\overline{1}$ Escuela Nacional de Antropología e Historia, México. mundosubterraneo@hotmail.com 


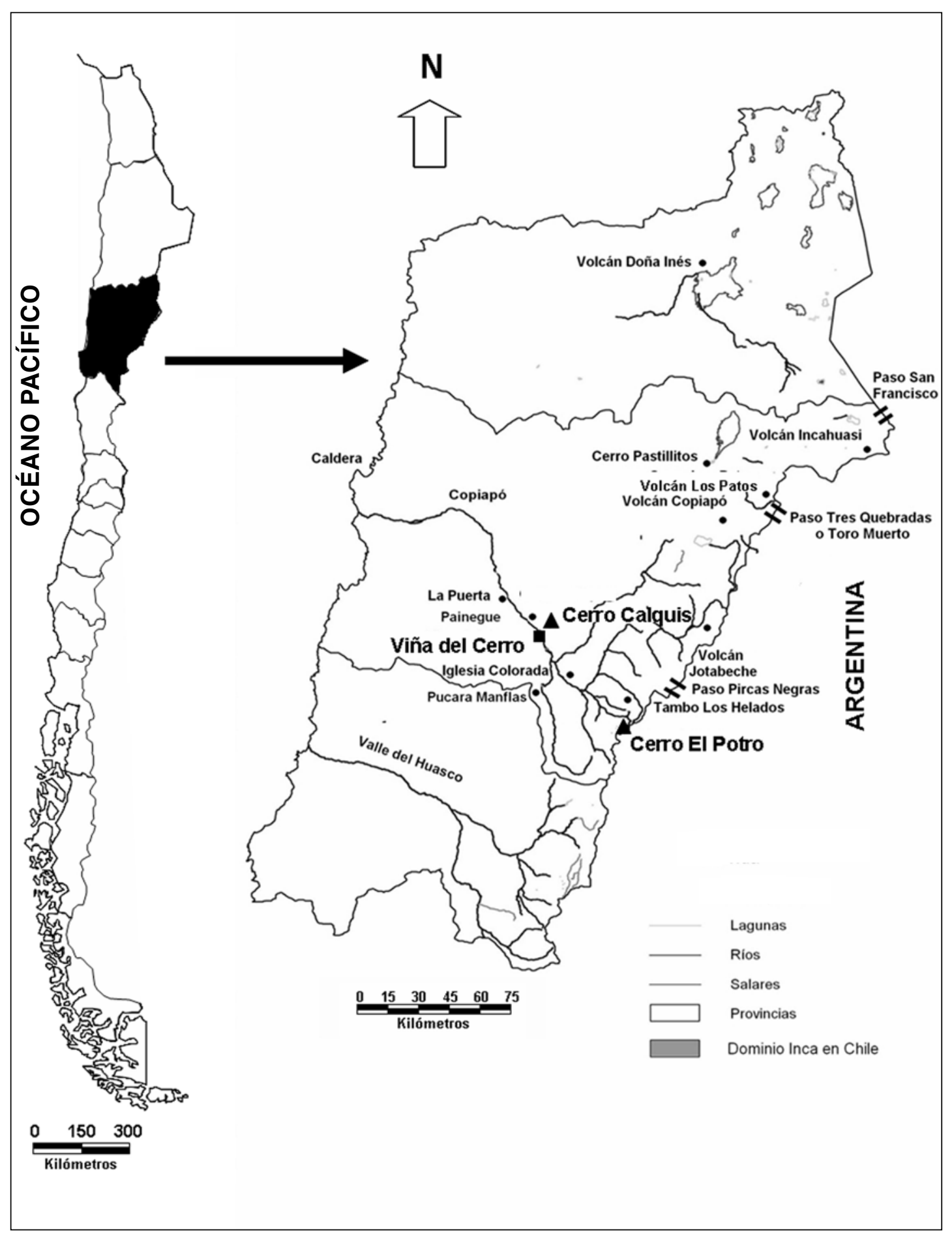

Figura 1. Mapa ubicación general.

Map of general location. 


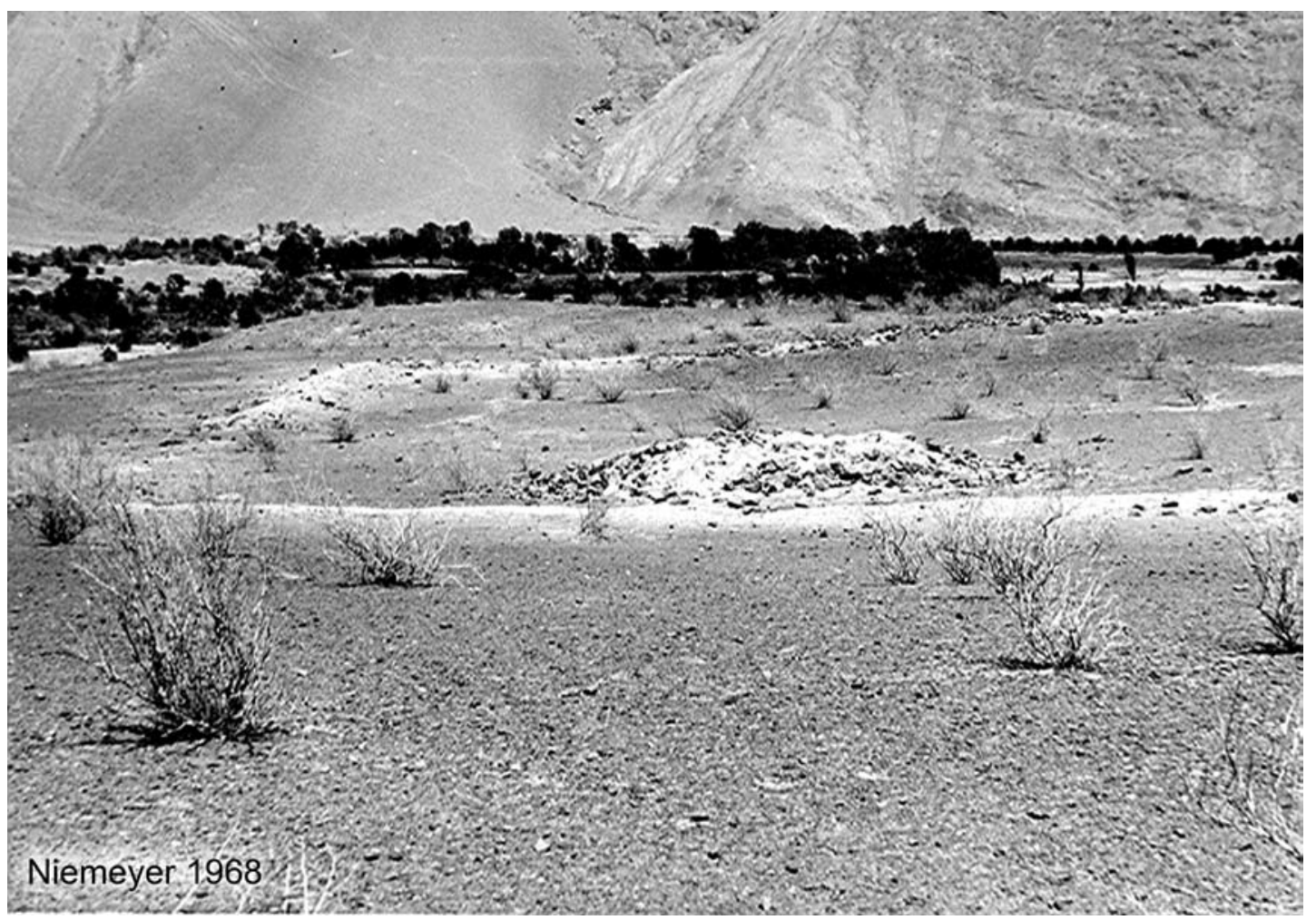

Figura 2. Ushnu y unidad A centro metalúrgico Viña del Cerro, 1968.

Ushnu and Unit A metallurgical center of Viña del Cerro, 1968.

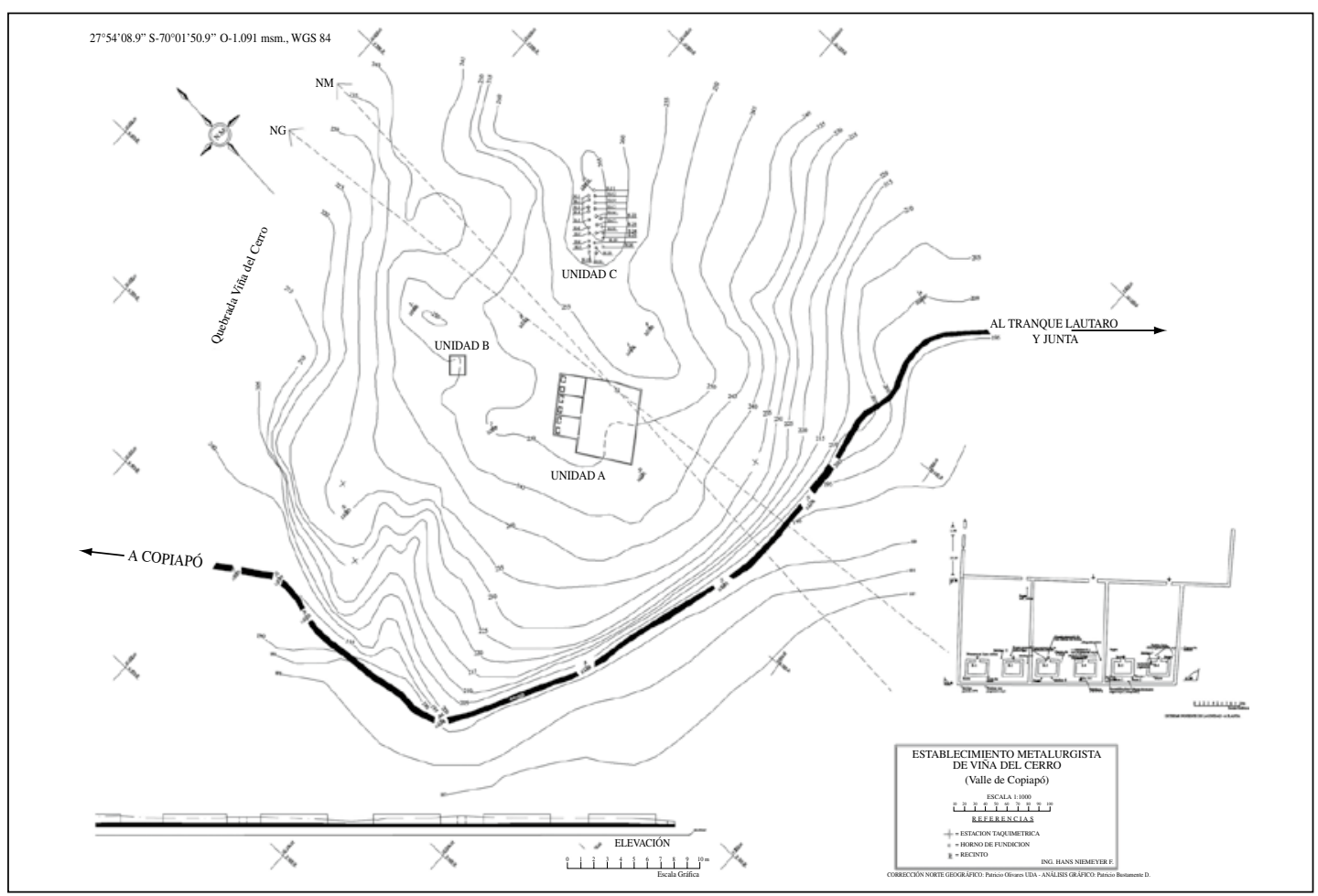

Figura 3. Levantamiento topográfico Viña del Cerro (Niemeyer 1979-1980).

Topographical survey of Viña del Cerro (Niemeyer 1979-1980). 
en el origen y desarrollo de las culturas andinas. Por estas razones creemos necesario ampliar el concepto del ushnu, no limitándolo sólo a la plataforma, para lo cual incluimos el análisis de las variables astronómicas y topográficas, con el objetivo de encontrar posibles relaciones entre la orientación acimutal de la arquitectura del centro metalúrgico ${ }^{2}$, la posición del Sol en el horizonte para fechas de solsticios y equinoccios, la relación con determinados elementos de la topografía local, así como su posible relevancia calendárica a partir de la comparación con otros sitios de similares características (véase Boccas 2004; Bustamante 2006; Pino 2004, 2005; Stehberg 2006).

\section{El Centro Metalúrgico Viña del Cerro}

El centro metalúrgico Viña del Cerro se compone de cuatro unidades arquitectónicas (Figura 3). (1) Un conjunto de 26 huayras u hornos de fundición ubicadas en el sector más ventoso y alto del emplazamiento (unidad C). Las huayras se distribuyen en dirección SW a NE, fueron construidas en un emplantillado de piedras y adobe, habrían tenido a lo menos $30 \mathrm{~cm}$ de altura, formas circulares y elípticas, diámetros que variaban de los 2 a $3 \mathrm{~m}$, y al parecer carecieron de una sección aérea permanente (Niemeyer 1986:178).

(2) Un puesto de control destinado seguramente a funciones administrativas (unidad B), compuesto de una pequeña kancha rectangular construida en barro y piedra, con muros de $15 \times 16 \mathrm{~m}$ de largo y $60 \mathrm{~cm}$ de ancho, y un vano de acceso de 1,2 $\mathrm{m}$ en el lado sureste. En su interior presenta un recinto de planta rectangular de 3,2 $\mathrm{m}$ de largo y $2 \mathrm{~m}$ de ancho, muros de $60 \mathrm{~cm}$ de ancho y vano de acceso en el lado suroeste (Castillo 1997). (3) Una pequeña estructura de piedra ubicada junto a una vertiente de agua dulce que emana del cerro Calquis (unidad D), con dimensiones originales de $6 \times 4 \mathrm{~m}$, no dibujada en el plano (Niemeyer 1986:178). Aquí es posible identificar algunas piedras semicanteadas distribuidas sin un orden lógico, mineral de cobre, abundante cerámica monocroma, Copiapó Negro sobre Rojo, Punta Brava y Diaguita-Inka (Moyano 2006:24). (4) Por último, la unidad A se ubica en el sector más plano del espolón rocoso. Corresponde a una kancha de 58,5 $\times 52 \mathrm{~m}$ con muro perimetral de piedra, adobe y barro que comprende dos conjuntos arquitectónicos: el campamento y la plataforma interpretada por Niemeyer como el ushnu. El campamento se localiza en el extremo noroccidental de la kancha y corresponde a un espacio rectangular de $48 \times 20,3 \mathrm{~m}$ construido con piedras esquinadas semicanteadas, adobes y argamasa de barro. Este espacio se divide por dos muros transversales en tres rectángulos de dimensiones iguales e independientes, comunicados por sus respectivos vanos al patio principal o kancha. En el extremo noroeste de cada uno de estos espacios existen dos recintos con dimensiones promedio de $2,3 \times 3,4 \mathrm{~m}, 70$ a $90 \mathrm{~cm}$ de altura y 60 a $70 \mathrm{~cm}$ de ancho en sus muros, con vanos de acceso al sureste de $60 \mathrm{~cm}$. Para la construcción de los recintos, así como para el muro perimetral, se usaron piedras esquinadas traídas de los cerros cercanos y adobones de barro calzado con argamasa (Niemeyer 1986:177). Hoy los muros presentan una altura promedio de 2,3 $\mathrm{m}$ (trabajo de restauración), además de un deterioro evidente por agentes antrópicos y naturales. Por su parte, la plataforma identificada como un ushnu se ubica en el rincón este del gran patio o kancha, tiene una forma de planta trapezoidal con muros de $6 \mathrm{~m}$ de largo en promedio, construidos en barro y piedra. La plataforma presenta una escalinata de 1,4 m de ancho y siete peldaños ubicados en un extremo del muro suroeste. Las excavaciones permitieron deducir que originalmente para la construcción de ésta se siguió un proceso parecido al de los recintos de la unidad A. Éste consiste en cerrar completamente un espacio mediante un muro perimetral de piedras esquinadas, espesor medido en el muro noreste de $90 \mathrm{~cm}$ y en el muro noroeste de $1,2 \mathrm{~m}$, el que luego se relleno con adobones y piedras (Niemeyer 1986:177-178). Nuevos antecedentes revelan que la plataforma sirvió como lugar para el entierro de un ser humano de tiempos históricos, y que posiblemente estuvo vinculada a través de un sistema de drenaje, suponemos subterráneo, con un pequeño vano ubicado en el muro suroeste del gran patio (Moyano 2005, 2006, 2007). De la cerámica recuperada de la plataforma durante la excavación, sabemos que casi en su totalidad representa el tipo local Punta Brava. Entre los materiales no se encontraron fragmentos de cerámica Copiapó Negro sobre Rojo o Ante o Diaguita-Inka. Esto podría suponer funciones domésticas, p.ej. la existencia de grandes contenedores de líquidos; o bien, que este lugar al igual que otros sitios tardíos de la región fuera construido sobre un asentamiento preincaico o Copiapó (Moyano 2006:174) ${ }^{3}$. 


\section{El Concepto Ushnu}

El ushnu ha sido descrito generalmente como plataformas al interior de grandes plazas o espacios de carácter público en el Cusco y en algunas de las principales capitales provinciales o Tampus del Tawantinsuyu. Estas plataformas fueron construidas en piedras y barro, presentan formas cuadrangulares, rectangulares o de pirámide trunca, tienen orientación cardinal o en ángulo, es decir, sin un patrón regular, y en su mayoría exhiben o habrían tenido escaleras, terrazas, tianas y cochas asociadas a sistemas de drenaje subterráneo (Gasparini y Margolies 1977; Hyslop 1990). Geográficamente estas plataformas se distribuyen entre Tomebamba y Mirador de Mullupungo en el Ecuador por el norte (Odaira 1998) y pukara de Chena en Chile Central por el sur (Boccas 2004). Sin embargo, las más conocidas por su monumentalidad arquitectónica son las plataformas de Huanuco Pampa, Pumpu, Tambo Colorado, Vilcas Huaman, Usno Moq'o y Cajamarca en Perú (Gasparini y Margolies 1977; Hyslop 1987, 1990, 1992; Matos 1994, 1995; Meddens 1997; Morris 1987; Oberti 1997). Inclusive se sugiere que los Intiwatanas de Machu Picchu, Tipon y Pisaq pudieran constituir algún tipo de ushnu por sus características de gnomon (Hyslop 1990:73). En los Andes Meridionales la presencia de estas plataformas ha sido definida como un rasgo arquitectónico de primer orden o incaico (Raffino 1981). Estos son los casos de Inkallajta de Cochabamba, La Fortaleza, Oma Porco de Aullagas, Chagua de Talina, Potrero de Payogasta de Calchaquí, Ingamana de Yocavil, Nevados de Aconquija, Hualfín, El Shincal, Watungasta de Abaucán, Tambería del Inka en Chilecito, Chaquiago de Andalgalá y Ampajango 2, distribuidos entre el sur de Bolivia y el noroeste de Argentina (Raffino 1981, 1993 [1991]; Raffino et al. 1985; 1997; Tarragó y González 2005). En Chile además de la plataforma del centro metalúrgico Viña del Cerro existen otras cinco con similares características. Éstas se ubican en tambo Chungará o "Tambo Quemado" en el altiplano de Arica (Chacón y Orellana 1979), Pampa Alto Ramírez (AZ-15) a $8 \mathrm{~km}$ al interior la misma ciudad (Santoro y Muñoz 1981), Saguara en Tarapacá (Schiappacasse y Niemeyer 2002), Cerro Verde en Atacama (Adán y Uribe 2005) y Chena al sur de Santiago (Boccas 2004). Estas plataformas no han sido estudiadas desde el punto de vista del calendario o el paisaje, a excepción de la última, donde se propone la existencia de líneas de visión hacia elementos de la topografía, p.ej. cerro El Plomo, cordón Provincia-San Ramón, principalmente para el solsticio de junio y el inicio de la temporada de lluvias en Chile Central (Boccas 2004; Bustamante 2006; Stehberg 2006). En general, se postula que estas plataformas estarían vinculadas con la red vial incaica o Qhapaq Nan, y responderían a la necesidad económica y política del Tawantinsuyu por controlar los recursos mineros de la parte sur del imperio. La primera referencia de la palabra ushnu la entrega Santo Tomás en su diccionario de 1560, donde define $O z \tilde{n} o$ u osño, como "altar, para sacrificar, altar donde sacrifican". Luego en 1568 Cristóbal de Albornoz en su lista sobre las huacas del Cusco identifica la palabra Usno como el lugar donde el Inka literalmente bebía al Sol. En esta interpretación el Usno habría cumplido funciones de gnomon, para los pasos cenitales, y también como huaca en los caminos y plazas de las provincias del Tawantinsuyu (Zuidema 1989:402-454). Sabemos también que el ushnu habría cumplido un rol político y público durante la fiesta del mes lunar de septiembre o Cituaquilla. En este mes, según el Cronista Anónimo (1906 [ca. 1570]), en Cusco se reunían los incas en la plaza de Haucaypata, donde se realizaban sacrificios al Sol desde un "teatro llamado Osno" (citado en Zuidema 1980:326-327). En 1608 González Holguín define usnu como "tribunal de juez de una piedra hincada; mojón cuando es de piedra grande hincada; altar antiguo" y la palabra usno, como lugar "para los sacrificios; montón de piedras". Mientras Bertonio en 1612 define husnu como "altar de los guacas hechos de piedras labradas, como se ve en las punas" (Zuidema 1989:445-446). En 1653 el padre Bernabé Cobo en su "Relación de los Adoratorios del Antiguo Cuzco" identifica con la palabra vsno a la primera huaca del quinto ceque de Antisuyu ubicada en la plaza de Haukaypata en Hurin Cuzco, donde "la primera era una piedra llamada, vsno, que estaua en la plaça de Hurin aukaypata; era esta la primera Guaca a quien ofrecian los que se hacian orejones" (Rowe 1981:232). Mientras Bartolomé de Segovia (1943 [1552]) refiere al ushnu como "un cuadro alto con terraplén, con una escalera muy alta" (citado en Rowe 1981:256). Cieza de León, aun cuando no utiliza la palabra ushnu, describe lo que a él le pareciera ser un "pan de azúcar" con funciones militares, "En la gran plaza de la ciudad del Cusco estaba la piedra de guerra, que era grande, de la 
forma y hechura de un pan de azúcar, bien engastonada y llena de oro" (Señorío, Cáp. XXIII, 1967:80, en Rowe 1981:256). Zuidema (2009) a partir de la lectura combinada de los cronistas Pizarro, Betanzos, Anónimo y Molina sugiere que el ushnu habría tenido tres componentes: un pilón (imagen del sol), una plataforma y posiblemente un pilar (agujero) ${ }^{4}$. En la ciudad del Cusco habrían existido al menos dos ushnus: uno ubicado al norte de la ciudad en la plaza de Hanan-Cuzco cerca del edificio del Sunturhuasi, y el otro al sur en la plaza de HurinCuzco. Cada ushnu y el Sunturhuasi, en su conjunto, habrían permitido realizar observaciones astronómicas en el horizonte central este y oeste para los días del paso del Sol por el cenit ${ }^{5}$ (octubre $30 \mathrm{y}$ febrero 13). Y por oposición de $180^{\circ}$ de la sombra proyectada por el gnomon, conocer con exactitud los días del paso del Sol por el anticenit ${ }^{6}$ (agosto 18 y abril 23), gracias a la existencia de cuatro pilares sobre el horizonte oeste (sucanca) que eran observados desde "otro pilar en medio de la plaça, pilar de piedra muy labrada, de un estado de alto ( $\sim 2 \mathrm{~m}$.), en un paraje señalado al propósito, que le nombrauan Osno" (Anónimo 1906:151, en Zuidema 2009, Apéndice 1). Estas fechas marcan el inicio y el fin de la temporada agrícola en Cusco, y coinciden con el período del año, cuando por efecto de la inclinación de la eclíptica ${ }^{7}$ es posible observar una Luna llena pasando por el meridiano muy cerca del cenit a medianoche (Zuidema 1980:320-321). De ahí que Zuidema (1989:402-454) vincule al ushnu con la acción de chupar (beber grandes cantidades de bebidas alcohólicas), los fenómenos meteorológicos y el inframundo. Y también con las observaciones astronómicas que eran realizadas desde cerro Sucanca hacia Quispicancha el 30 de octubre, para el inicio de la estación lluviosa o pucuy sucanca; y con la observación de la puesta del Sol para el anticenit (26 de abril), realizadas desde Quispicancha hacia cerro Sucanca, que marcaban el inicio de la estación seca o chirao sucanca (Zuidema 2008). Evidencia etnoarqueológica concuerda con estos trabajos e indica una fuerte relación entre el ushnu, las actividades agrícolas y observaciones astronómicas. Se señala que los ushnus estarían "relacionados con el agua que baja del cielo a las montañas, es decir, con un culto a la fertilidad [donde] los ushnu actuarían como reguladores del clima" (Meddens 1997:7). Así como con la ceremonia de la capac hucha, ciertas libaciones, el culto a los ancestros y las montañas, sistemas de drenaje, el inframundo y con la idea de centro en las provincias conquistadas (Meddens et al. 2008; Staller 2008). Pino hace hincapié en este hecho y sugiere un origen preincaico del concepto ushnu en la Sierra Central del Chinchaysuyu (Perú), donde este vocablo se vincula con huecos en el subsuelo, lugares donde se encuentran los muertos y los gentiles, ruinas peligrosas donde salen enfermedades, pozos de agua, lugares con muchos cascajos y donde se filtra el agua, e inclusive con las plataformas de los cerros altos (Pino 2005:146). Según el mismo autor, este concepto habría sido incorporado por los incas dentro de su política expansiva, incluyendo luego la plataforma, la que pasó a ser el centro en la planificación arquitectónica (radial-astronómica) de los principales Tampus del Tawantinsuyu, p.ej. Huanuco Pampa, Pumpu, Inkawasi, Tambo Colorado y Vilcas Huaman (Pino 2004, 2005). Aquí se propone un modelo que divide el espacio arquitectónico en NE, SE, NW y $\mathrm{SW}$, cada uno con cuatro direcciones astronómicas (incluidas el norte y sur verdadero), y la división geométrica del espacio a partir del equinoccio solar. Un total de 20 direcciones que toma en cuenta la posición del Sol para los solsticios y pasos cenitanticenit, salida y puesta del conjunto Alfa y Beta Centauro/Cruz del Sur (y su inversa), así como la salida y puesta de la Luna en sus posiciones extrema norte y sur (Pino 2005:149-150).

\section{El Método de Investigación}

Esta investigación involucró la observación y registro de fenómenos astronómicos a simple vista con ayuda de una brújula magnética, con precisión de un $1^{\circ}$, desde la plataforma identificada como un ushnu (in situ), para fechas de solsticios y equinoccios (2004-2006), junto con el reconocimiento arqueológico de las zonas identificadas en el horizonte para el movimiento anual del Sol y elementos significativos del paisaje (Moyano 2006:148-169). En un primer momento se utilizaron los planos originales de Viña del Cerro realizados por Hans Niemeyer entre 1979 y 1980, pero luego fue necesario elaborar un nuevo plano general de la unidad A y la plataforma que formaba parte del ushnu, en octubre del año 2005 (Figura 4). Para la determinación del norte geográfico o verdadero se realizaron observaciones solares con un taquímetro análogo PENTAX TH-60E, junto con los valores entregados por una calculadora de Estimación del 


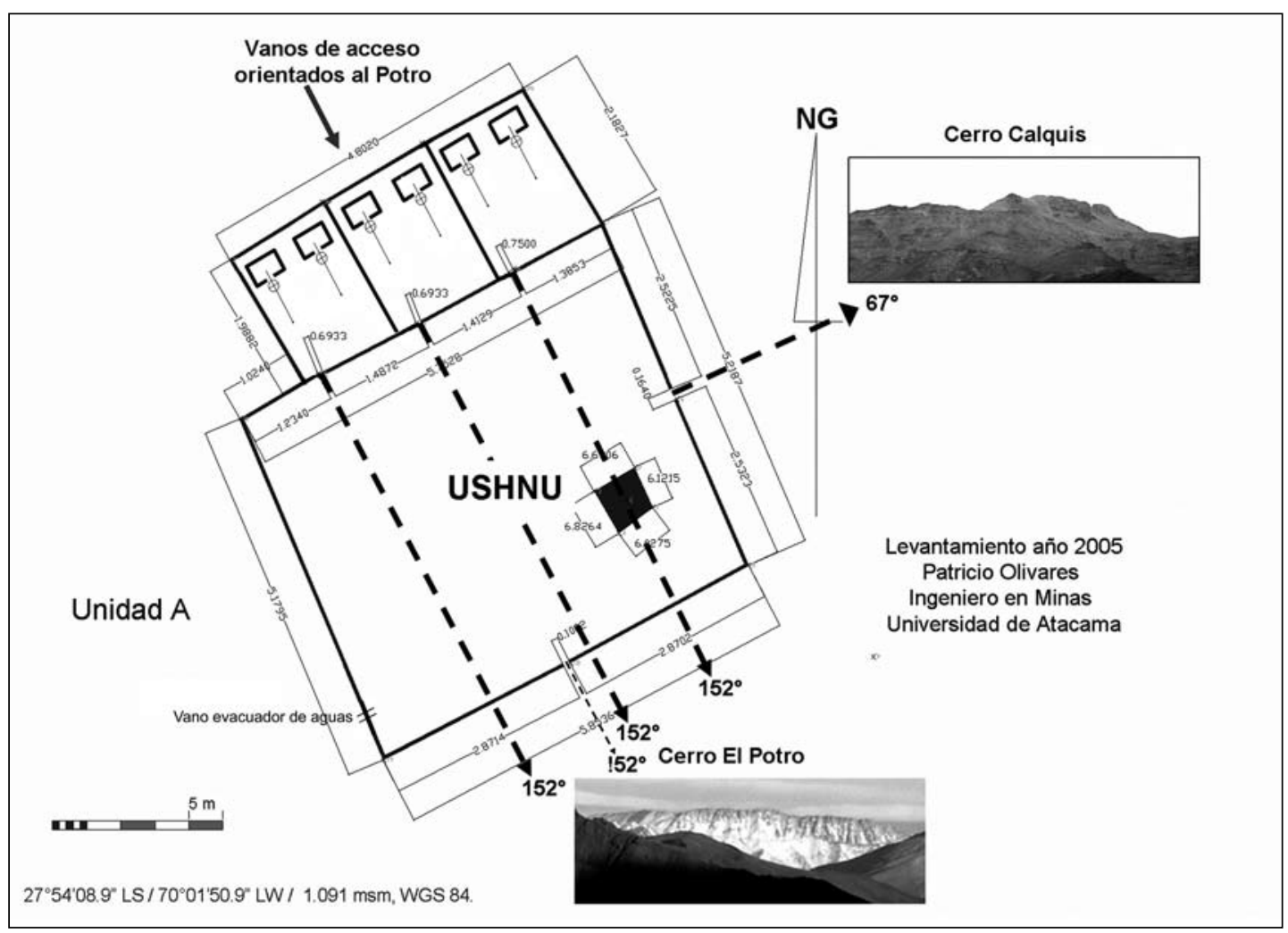

Figura 4. Unidad A centro metalúrgico Viña del Cerro. Unit A, metallurgical center of Viña del Cerro.

Valor de la Declinación Magnética ${ }^{8}$. El acimut o distancia angular desde el norte verdadero, de salida y puesta del Sol, la Luna y determinadas estrellas sobre el horizonte teórico, se obtuvo gracias a los datos entregados por el astrónomo Fernando Noel y el uso de los software planetarios Horizons Ephemerides Generador del Jet Propulsión Laboratory-NASA y Redshift 5 Maris Technologies USM. El análisis gráfico de la información se realizó sobre una Carta Solar, o representación gráfica de la trayectoria anual del Sol (acimut y altura) para la ciudad de Copiapó (Stuven 1972). Se utilizó además una fotografía panorámica de $360^{\circ 9}$ tomada desde la plataforma. Conocida la declinación magnética para nuestra posición geográfica $\left(27^{\circ} 54^{\prime} 08.9^{\prime \prime} \mathrm{LS}, 70^{\circ}\right.$ 01 ' 50.9" $\mathrm{LW}$ ) con valores que variaron entre $0^{\circ}$ $27^{\prime}$ E (junio 2004) y $0^{\circ} 11^{\prime}$ E (marzo 2006), en la fotografía panorámica se dejó el norte geográfico en el centro, para luego calar la imagen cada $2^{\circ}$ completando una fotografía de $360^{\circ}$, con el sur en los extremos. En esta fotografía se procedió a marcar los acimutes observados y teóricos, para solsticios y equinoccios, de la salida y puesta del Sol, y con ello la corrección gráfica de los acimutes observados en terreno ${ }^{10}$ (Figura 5). Con esta información se procedió a la representación gráfica de las orientaciones de muros y diagonales de la plataforma que podrían haber formado parte del ushnu (Figura 6), junto con los vanos de acceso de los recintos y muros de la unidad A, a partir del levantamiento topográfico realizado en octubre de 2005 (Figura 4). Finalmente se construyó una Carta Solar Entorno de la posición del Sol (solsticios y equinoccios), la Luna (posiciones extrema norte y sur) y el conjunto Alfa y Beta Centauro/Cruz del Sur ${ }^{11}$ sobre el horizonte matemático, es decir, sin tomar en cuenta la altura del horizonte, hacia el año 1.470 d.C., junto con indicar la posición de algunos cerros de la topografía local en una circunferencia de $360^{\circ}$, como una aproximación de las principales orientaciones identificadas durante el trabajo de campo (Figura 7). Se tomó como referencia el año de 1.470 d.C., considerando el movimiento de precesión $\left(1^{\circ}\right.$ cada 72 años $=2$ veces el diámetro 


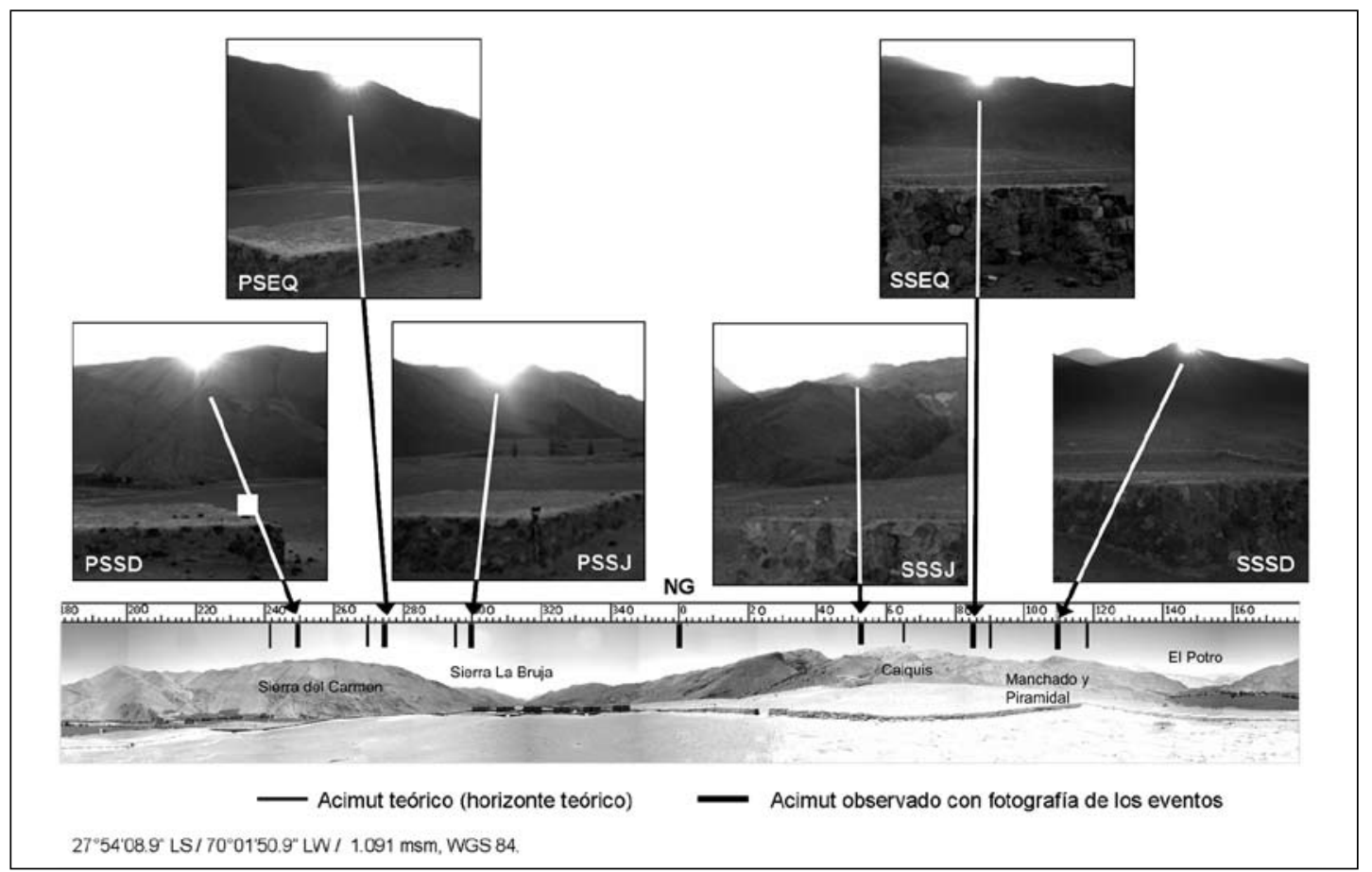

Figura 5. Fotografía panorámica 360 grados.

360-degree panoramic photograph.

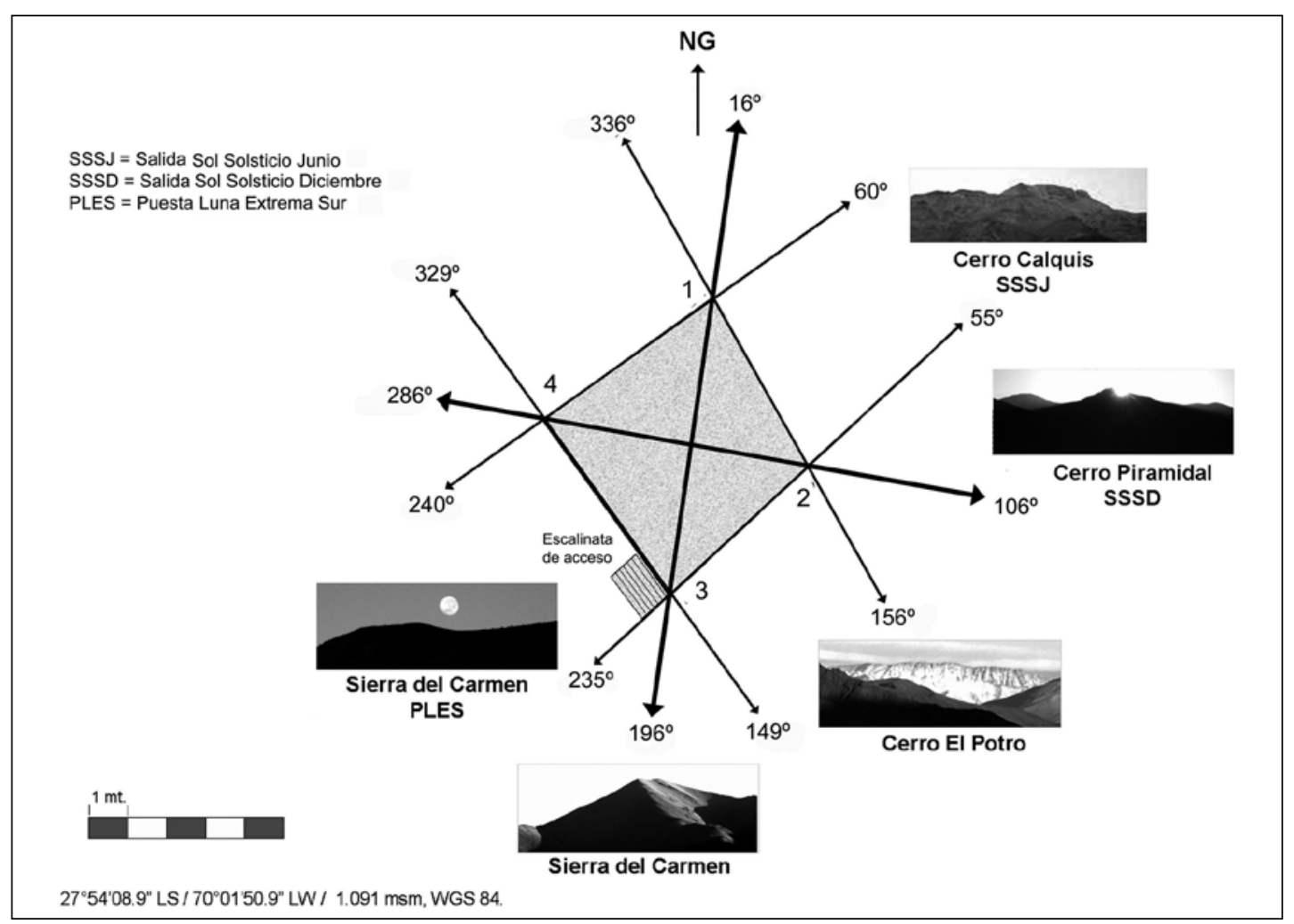

Figura 6. Ushnu centro metalúrgico Viña del Cerro.

Ushnu, metallurgical center of Viña del Cerro. 


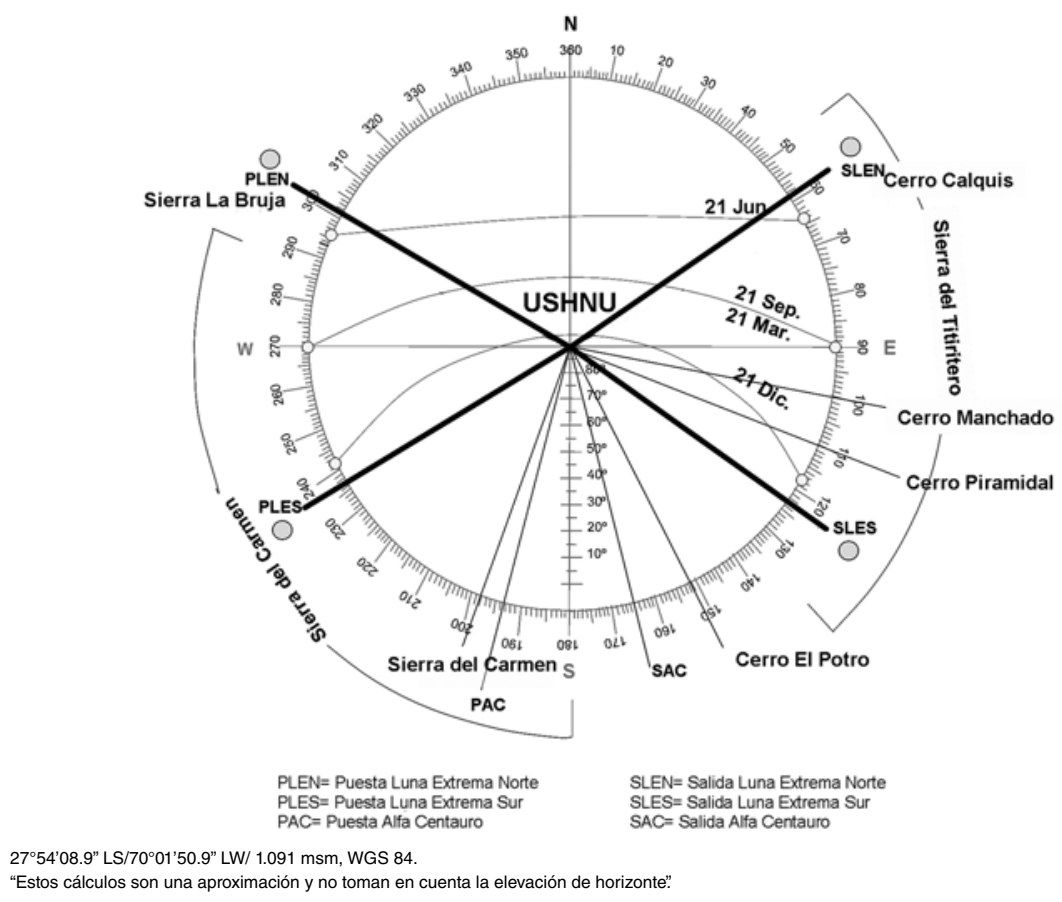

Figura 7. Carta Solar Entorno Viña del Cerro.

Solar and surroundings diagram of Viña del Cerro.

aproximado de una Luna llena), y las fechas para la llegada del Tawantinsuyu al valle de Copiapó, posterior al año 1.400 d.C. (Castillo 1997).

\section{Análisis de la Información}

El análisis de las orientaciones acimutales de las diagonales y muros de la plataforma, levantamiento topográfico 2005 (Figura 6), entrega los siguientes resultados:

(a) Los tiros $1-2\left(\right.$ acimut $156^{\circ}$ ) y $4-3$ (acimut $149^{\circ}$ ) estarían orientados al sureste y sector del valle de Copiapó dominado por el macizo andino El Potro $(5.886 \mathrm{msm})$. Esta montaña se ubica en las cabeceras del río Copiapó, donde gracias a la existencia del glaciar Los Helados se sostiene el sistema hídrico de la cuenca. Trabajos realizados en la zona desde la década de 1970 dan cuenta de sitios arqueológicos de tipo ceremonial y parte del sistema vial incaico en la zona, lo que sustenta la idea de un cerro huaca en la cabecera del río Copiapó (Beorchia 1985; Niemeyer 1986; Moyano 2006, 2008). Esto podría resultar relevante, si consideramos las relaciones de intervisibilidad existentes entre otras huacas y ushnus incaicos, principalmente en las tierras altas de Ayacucho, Perú (Meddens et al. 2008), y el ushnu de Chena al sur de Santiago (Boccas 2004; Bustamante 2006; Stehberg 2006).

(b) Los tiros 3-2 (acimut $55^{\circ}$ ) y 4-1 (acimut $60^{\circ}$ ) se orientan a la salida del Sol para el solsticio de junio (SSSJ) y un sector muy cercano a la cumbre del cerro Calquis. Calquis o Kalka es un topónimo quechua para "lugar pedregoso o lleno de piedras" (Soto 1976:55). Que en un caso podría hacer referencia a la idea de ushnu recogida por Pino (2004, 2005) en los Andes peruanos, o también a las concentraciones de mineral de cal en las laderas del cerro Manchado en las cercanías de Calquis ${ }^{12}$. El reconocimiento arqueológico de la zona, aun cuando no entregó resultados positivos, deja abierta la posibilidad a que esta montaña fuera un adoratorio local en tiempos prehispánicos, considerando la existencia de minas de cobre en la zona y el fuerte tono rojizo que la misma adquiere durante los atardeceres, que constituyen un punto de referencia en la topografía, 
más que astronómico desde el punto de vista de Viña del Cerro.

(c) La diagonal 4-2 (acimut $106^{\circ}$ ) se orienta hacia un sector muy cercano a la cumbre con forma de V invertida o piramidal (contrafuerte de Calquis), por donde sale el Sol para el solsticio de diciembre (SSSD). En este lugar se identificó una pequeña apacheta de no más de $50 \mathrm{~cm}$ de altura, ubicada junto a un sendero utilizado por mineros y arrieros locales (Moyano 2006). Aun cuando ésta podría ser de construcción moderna, consideramos sugerente la existencia de la misma, si tomamos en cuenta la presencia de una animita católica a los pies del mismo cerro y la forma de la montaña vista desde Viña del Cerro, que podría haber servido como un marcador natural de horizonte para el solsticio de diciembre.

(d) La diagonal 1-3 (acimut $196^{\circ}$ ) se orienta hacia una de las cumbres más altas y llamativas de la Sierra del Carmen, primera en recibir los rayos del Sol durante los amaneceres (marcador orográfico). Este topónimo podría estar vinculado con un culto prehispánico a la Pachamama y, orográficamente hablando, con el sur astronómico y la posición del conjunto Alfa y Beta Centauro/Cruz del Sur sobre el horizonte (Figura 7).

(e) $\operatorname{Los}$ tiros 2-3 (acimut $235^{\circ}$ ) y 1-4 (acimut $240^{\circ}$ ) se orientan al suroeste y la Sierra del Carmen muy cerca de donde debiéramos observar la puesta de una Luna llena en su posición extrema sur $-28.36^{\circ}$ (PLES) o detención lunar mayor. Este lunisticio ocurre cada 18,61 años, y en teoría podría haber sido conocido por los constructores y/o ocupantes de Viña del Cerro considerando la latitud geográfica del valle de Copiapó cercano al trópico lunar meridional y la importancia que tiene la Luna dentro de los calendarios andinos prehispánicos (Ianiszewski, comunicación personal 2006).

El análisis general de la arquitectura de la unidad A (Figura 4) y del horizonte (Figura 5) en Viña del Cerro entregó los siguientes resultados:

(a) Los vanos de acceso a los patios secundarios, los vanos de los recintos del campamento y uno de los vanos de acceso a la kancha se orientan en dirección sureste con un acimut de $152^{\circ}$ al cerro El Potro, donde se constató la existencia de una posible huaca incaica (Beorchia 1985; Niemeyer 1986; Moyano 2006, 2008).

(b) El vano que da acceso a la kancha desde el noreste se orienta con un acimut de $67^{\circ}$ (perpendicular a la pared) a la cumbre del cerro Calquis, muy cerca de la salida del Sol para el solsticio de junio (SSSJ), fecha importante dentro del calendario andino (Zuidema 1995 [1964]), además del límite físico en el desplazamiento del Sol hacia el norte.

(c) Desde el punto de vista de la astronomía de horizonte, la observación de los solsticios desde el ushnu de Viña del Cerro (Figura 5) indica que era posible conocer el inicio del verano en el hemisferio sur entre el 21 y el 24 de diciembre gracias a la observación de una salida de Sol, por un contrafuerte del cerro Calquis en forma de V invertida o piramidal (SSSD), acimut $110^{\circ}$. Junto con ello y en oposición de $180^{\circ}$, conocer el inicio del invierno en el hemisferio sur entre el 21 y el 24 de junio gracias a la observación de una puesta de Sol en un portezuelo en forma de U en las laderas de la Sierra La Bruja (PSSJ), acimut $300^{\circ}$. En este último lugar se constató la existencia de al menos un par de apachetas, no visibles desde la posición de Viña del Cerro, que estarían marcando la ubicación de una pequeña mina de cobre en la zona (Moyano 2006).

(d) En lo que refiere a la observación de los equinoccios (marzo y septiembre), la salida del Sol ocurre en las laderas del Calquis, pero en ningún marcador natural de horizonte. Sin embargo, los ocasos para estas dos fechas suceden en la Sierra del Carmen con un acimut de $273^{\circ}$, lugar que por resultado del juego de luz y sombra pareciera observarse el perfil de un rostro humano (Moyano 2006:152). Una inspección de la zona permitió conocer la existencia de un socavón minero de cobre con presencia de cerámica estilo La Puerta (600900 d.C.) y fragmentos de vidrio del siglo XIX. Una explicación a la identificación de formas conocidas, p.ej. rostros humanos o de animales, en el paisaje nos la entrega Bustamante (2008), quien propone a los fenómenos psicológicos de la Pareidolia (identificación automática de una semejanza formal); la Apofenia (establecimiento de relaciones complejas entre fenómenos no directamente relacionados), y la Hierofanía (identificación de formas naturales conocidas y las subsecuentes relaciones 
con hechos numinosos o de manifestación divina) como una de las tantas razones para la sacralización del espacio en tiempos prehispánicos. Más aún, trabajos en las cercanías de OllanTaytambo, Perú, indican una fuerte relación entre diseños antropo y zoomorfos, (arquitectura y componentes del paisaje) con eventos astronómicos en sitios incaicos (Elorrieta y Elorrieta 1996).

(e) No se descarta la posibilidad de que el eje de construcción de las huayras (unidad C) orientación noreste-suroeste (salida Sol solsticio junio-puesta Sol solsticio diciembre), y que el número de las mismas $(\mathrm{N}=26)$, cercano pero no igual a la extensión del calendario lunar sideral (27,3 días) (Zuidema 2009), pudiera tener alguna correlación astronómica y calendárica $^{13}$, así como también las seis unidades residenciales (unidad A), distribuidas de a par en tres patios secundarios y orientadas hacia el cerro El Potro, que podrían simbolizar los principios andinos de dualidad, tripartición y adoración a los ancestros.

\section{Consideraciones Finales}

Los ushnus se han vinculado con desfiles militares, tribunales de justicia, la política, rituales como la capac hucha y la Citua, el culto a la fertilidad y los antepasados, la observación astronómica y centro para la planificación arquitectónica de las capitales provinciales del Tawantinsuyu. En el caso de Viña del Cerro, el análisis de las orientaciones acimutales de la arquitectura reconstruida de la plataforma y unidad A sugiere que este lugar, además de funciones políticas y administrativas vinculadas con la contabilidad y la producción metalúrgica de minerales de cobre y plata, habría servido también como escenario para la realización de actividades públicas vinculadas con el culto solar y a las montañas, ambas de amplia distribución geográfica y temporal en los Andes. Aquí la construcción de un ushnu pudo responder a la necesidad de los incas por resaltar condiciones de la topografía local preexistente. Estas incluyeron: Primero, la posición geográfica estratégica del centro metalúrgico cercana a numerosos yacimientos mineros, rutas de tráfico en sentido norte-sur y este-oeste. Segundo, los fuertes vientos que bajan desde la cordillera de los Andes necesarios para las actividades metalúrgicas. Tercero, su ubicación privilegiada sobre un espolón rocoso que permitió el control de las rutas caravaneras por el valle de Copiapó, y también congregar a un número importante de personas en el sector de la kancha. Cuarto, la visibilidad permanente de las montañas Calquis y El Potro que pudieron ser consideradas sagradas por su relación con los fenómenos meteorológicos y los ritos de fertilidad. Quinto, la existencia de "marcadores naturales de horizonte" para la puesta del Sol en el solsticio de junio y la salida del Sol en el solsticio de diciembre (PSSJ y SSSD), lo que habría permitido conocer la extensión del año solar y organizar la producción metalúrgica local a partir de la recurrencia de eventos cíclicos. Desde un punto de vista comparativo (véase Pino 2004, 2005), el sistema de orientaciones en Viña del Cerro resulta mucho más simple y acotado que el de Huanuco Pampa y otros Tampus del Chinchaysuyu, resultado de la importancia simbólica y/o práctica del centro metalúrgico, orientado más a la producción de mineral de cobre que al control de un calendario regional. Sin embargo, como atestiguan los trabajos de Meddens y colaboradores en Ayacucho (2008), así como los de Boccas (2004), Bustamante (2006) y Stehberg (2006) al sur de Santiago, creemos en el potencial interpretativo (etnográfico y arqueológico) con respecto a la relación entre el ushnu de Viña del Cerro y determinados elementos de la topografía, p.ej. los cerros Calquis y El Potro. A estos elementos de análisis se agrega la ubicación geográfica del valle de Copiapó, al sur del desierto de Atacama y del trópico de Capricornio, donde no ocurren pasos cenitales solares, adquiriendo mayor importancia la observación de los solsticios y quizás la Luna junto con algunas constelaciones que nos indican el polo sur celeste, como el conjunto Alfa y Beta Centauro/Cruz del Sur. Resulta interesante además la presencia de un pequeño vano (evacuador de aguas) en el muro suroeste de la kancha (elemento ctónico), que sugiere la existencia de los elementos del ushnu identificados en el Cusco por Zuidema (1980, 1989, 2009). En este caso la plataforma podría haber funcionado como gnomon, mientras que el agujero o "pilar" eventualmente se habría ubicado cerca del centro de la plaza donde se interceptan las líneas de visión de los cerros Calquis y El Potro (Zuidema, comunicación personal 2007). Tomando en cuenta estos antecedentes, debiéramos considerar al ushnu en Viña del Cerro como un concepto abstracto, que podía o no incluir una o todas las características formales identificadas por Zuidema y Pino en sus trabajos. En otras palabras, 
no necesariamente un centro para la planificación arquitectónica del emplazamiento, pero sí el lugar donde se conectaron todas las direcciones sagradas del espacio-tiempo o pacha en un determinado espacio ritual y social: el Hanan pacha o mundo de arriba, representado por las deidades celestes y el Sol; el Kay pacha o mundo de acá, representado por la producción metalúrgica y la vida litúrgica en la kancha; y el Hurin pacha o mundo de abajo, representado por las riquezas del inframundo, en este caso los minerales, y la acción chupar o beber por parte de la tierra. Y en un sentido más político y hegemónico, el lugar que simbolizó la autoridad del Inka, la complementariedad y la reciprocidad andina, a través de la incorporación de los elementos potencialmente sagrados de la topografía local, junto con la elaboración y ajuste de un calendario solar de horizonte.
Agradecimientos: a los evaluadores externos, al Museo Regional de Atacama, al Departamento de Minas Universidad de Atacama, la Corporación Nacional Forestal (CONAF). A mis colegas María José Fernández, Francisco Garrido, Lorena Arancibia, Felipe Gutiérrez, Virginia Mcrostie, Pablo Reyes, Jorge Ianiszewski, Rodrigo Zapata, Dagoberto Lezcano, Michael Wolfe, Mario Huerta y Juan Carlos Carmona. A Miguel Cervellino y Gastón Castillo por la capacidad de gestión y consejos. A Eduardo Saavedra y Patricio Olivares por la elaboración y digitalización de los planos. A Fernando Noel por los cálculos astronómicos, a Patricio Bustamante por la elaboración de las figuras y a R.T. Zuidema por sus comentarios. A Victoria Castro y Silvia Quevedo por creer en la arqueoastronomía. Especialmente a mi familia por entender y compartir mis sueños.

\section{Referencias Citadas}

Adán, L. y M. Uribe

2005 El dominio inca en la localidad de Caspana: un acercamiento al pensamiento político andino (río Loa, Norte de Chile). Estudios Atacameños 29:41-66.

Beorchia, A.

1985 El enigma de los santuarios indígenas de alta montaña. Revista del Centro de Investigaciones Arqueológicas de Alta Montaña (CIADAM). Tomo 5. U.N.S.J. San Juan.

Boccas, $\mathrm{M}$.

2004 Topografía y astronomía: dos herramientas de apoyo en arqueología. Chungara Revista de Antropología Chilena Número especial Tomo II: 1037-1048.

Broda, J.

2004 [2003] La percepción de la latitud geográfica: otra dimensión de los estudios sobre calendarios mesoamericanos y arqueoastronomía. Etno y Arqueo-Astronomía en las Américas, Memorias del Simposio ARQ-13 del 51 Congreso Internacional de Americanistas, pp. 77-96, Santiago.

Bustamante, $\mathrm{P}$.

2004 Obras rupestres, paisaje y astronomía en el Choapa. Werken 5:133-138.

2006 Santiago del nuevo extremo ¿Una ciudad sin pasado? Diseño Urbano y Paisaje 9. (30 diciembre) http://www. ucentral.cl/dup/pdf/9_santiago_nuevo_extremo.pdf (15 mayo 2009).

2008 ¿Qué Parece? Como pregunta orientadora en el estudio de la Topografía Sagrada en la cultura Azteca. (16 marzo). http://www.rupestreweb.info/queparece.html (31 marzo 2008).

Castillo, G.

1997 Los periodos Intermedio Tardío y Tardío: desde la Cultura Copiapó al dominio Inca. En Culturas Prehistóricas de Copiapó, editado por H. Niemeyer y M. Cervellino, pp. 163-282. Museo Regional de Atacama, Copiapó.
Chacón, S. y M. Orellana

1979 El tambo Chungará. Actas del VIII Congreso de Arqueología Chilena: 247-256, Valdivia.

Elorrieta, F. y E. Elorrieta

1996 El Valle Sagrado de los Incas, Mitos y Símbolos. Sociedad Pacaritanpu Hatha, Cusco.

Gasparini, G. y L. Margolies

1977 Arquitectura Inka. Centro de Investigaciones Históricas y

Estéticas Facultad de Arquitectura y Urbanismo, Universidad Central de Venezuela, Caracas.

Grebe, M. y B. Hidalgo

1988 Simbolismo atacameño: un aporte etnológico a la comprensión de significados culturales. Revista Chilena de Antropología 7:75-97.

Hyslop, J.

1987 Inkawasi: el mundo en microcosmos. Cuadernos del Instituto Nacional de Antropología 12:47-71. Buenos Aires.

1990 Inka Settlement Planning. University of Texas Press, Austin.

1992 Qhapaqñan, el Sistema Vial Inkaico. Instituto Andino de Estudios Arqueológicos, Petróleos del Perú. Epígrafe S.A. Editores, Lima.

Iribarren, J.

1958 Arqueología en valle de Copiapó. Revista Universitaria 43 (22):167-195. Santiago.

Matos, M.R.

1994 Pumpu: Centro Administrativo Inka de la Puna de Junín. Editorial Horizonte, Lima.

1995 Los Inka de la Sierra Central del Perú. Revista de Arqueología Americana 8:159-189.

Meddens, F.M.

1997 Function and meaning of the usnu in the Late Horizon Peru. Tawantinsuyu 3:5-14.

Meddens, F., N. Branch, C. Vivanco, N. Riddiford y R. Kemp 2008 High altitude ushnu platforms in the department of Ayacucho Peru, structure, ancestors and animating 
essence. En Pre-Columbian Landscapes of Creation and Origin, editado por J.E. Staller, pp. 315-355. Springer, New York.

Morris, C.

1987 Arquitectura y estructura del espacio en Huanuco Pampa. Cuadernos del Instituto Nacional de Antropología 12:27-45. Buenos Aires.

Moyano, R.

2005 Arqueoastronomía en el valle de Copiapó, una aproximación desde el paisaje cultural (Informe práctica profesional). FONDECYT 1011006. Manuscrito en posesión del autor. 2006 Sol, Luna y Huacas en el Valle de Copiapó, el Ushnu de Viña del Cerro como lugar de Observación Astronómica y Culto a las Montañas en Atacama. Memoria para optar al título de arqueólogo. Facultad de Ciencias Sociales, Universidad de Chile, Santiago.

2007 The ushnu of Viña del Cerro as a site for astronomical observation and mountain worship in Atacama. Ponencia presentada en The 35th Annual Midwest Conference on Andean and Amazonian Archaeology and Ethnohistory, Southern Illinois University at Carbondale, Illinois, USA.

2008 El adoratorio prehispánico del cerro El Potro: arqueología de alta montaña en la cordillera de Copiapó, norte de Chile. Manuscrito en posesión del autor.

Niemeyer, $\mathrm{H}$.

1986 La ocupación incaica de la cuenca alta del río Copiapó (III Región de Atacama, Chile). Comechingonia Número Especial: 165-294.

Niemeyer, H., M. Cervellino y E. Muñoz

1983 Viña del Cerro, expresión metalúrgica inca en el valle de Copiapó. Creces 4 (4):50-57.

1984 Viña del Cerro: metalurgia Inka en Copiapó, Chile. Gaceta Arqueológica Andina 9:6-7.

Niemeyer, H., G. Castillo y M. Cervellino 1993 [1991] Estrategia de dominio Inca en el valle de Copiapó. Actas del XII Congreso Nacional de Arqueología Chilena, Tomo I: 333-371, Temuco.

Oberti, I.

1997 Investigaciones preliminares en Usno-Moq'o, Abancay. Tawantinsuyu 3:15-21.

Odaira, S.

1998 El mirador de Mullupungo: un aspecto del control inca en la costa sur del Ecuador. Tawantinsuyu 5:145-152.

Pino, J.

2004 El ushnu Inka y la organización del espacio en los principales Tampus de los Wamani de la Sierra Central del Chinchaysuyu. Chungara Revista de Antropología Chilena 36:303-311.

2005 El ushnu y la organización espacial astronómica en la Sierra Central del Chinchaysuyu. Estudios Atacameños 29:143-161.

Raffino, R.

1981 Los Inkas del Kollasuyu. Ramos Americana Editores, La Plata.

1993 [1991] Uso territorial y patrón de asentamiento Inka en el altiplano del Kollasuyu. Actas del XII Congreso Nacional de Arqueología Chilena, Tomo I: 293-316, Temuco.
Raffino, R., R. Alvis, L. Baldini, D. Olivera y M. Raviña 1985 Hualfin-El Shincal-Watungasta, tres casos de urbanización Inka en el NO argentino. Cuadernos del Instituto Nacional de Antropología 10:425-455. Buenos Aires.

Raffino, R., D. Gobbo, R. Vázquez, A. Capparelli, V. Montes, R. Iturriza, C. Deschamps y M. Mannasero 1997 El ushnu de El Shincal de Quimivil. Tawantinsuyu 3:22-39.

Rowe, J.

1981 Una relación de los adoratorios del antiguo Cuzco. Histórica V (2):223-243. Lima.

Santoro, C. e I. Muñoz

1981 Patrón habitacional incaico en el área de pampa Alto Ramírez (Arica Chile). Chungara 7:144-171.

Schiappacasse, V. y H. Niemeyer

2002 Ceremonial Inka provincial: el asentamiento de Saguara (Cuenca de Camarones). Chungara Revista de Antropología Chilena 34:53-84.

Soto, C.

1976 Diccionario Quechua, Ayacucho-Chanca. Ministerio de Educación, Lima.

Staller, J.E.

2008 Dimensions of place: the significance of centers to the development of Andean civilization: an exploration of the ushnu concept. En Pre-Columbian Landscapes of Creation and Origin, editado por J. E. Staller, pp. 269-311. Springer, New York.

Stehberg, R.

2006 En torno al simbolismo del pucara de Chena. Diseño Urbano y Paisaje 9. (30 diciembre) http://www.ucentral.cl/ dup/pdf/9_pucara_chena.pdf (15 mayo 2009).

Stuven, $\mathrm{H}$.

197242 Gráficos Solares para Ciudades de Chile y Argentina. Facultad de Arquitectura, Universidad de Chile, Santiago.

Tarragó, M. y L. González

2005 Variabilidad en los modos arquitectónicos incaicos. Un caso de estudio en el valle de Yocavil (noroeste argentino). Chungara Revista de Antropología Chilena 37:129-143.

Urízar, M.

2002 Periodo de contacto indígena: la colonización española en el valle de Copiapó (Informe de avance). FONDECYT 1000037. Manuscrito en posesión de la autora.

Zuidema, R.T.

1980 El ushnu. Revista de la Universidad Complutense 28:317-362.

1981 Inca observations of the solar and lunar passages through zenit and anti-zenit at Cuzco. En Archaeoastronomy in the Americas, editado por R. Williamson, pp. 319-342. Ballena Press, Los Altos.

1989 El ushnu. En Reyes y Guerreros: Ensayos de la Cultura Andina, compilado por M. Burga, pp. 402-454. Ediciones Fomciencias, Lima.

1995 [1964] El Sistema de Ceques del Cuzco. Traducido por E. Salazar. Pontificia Universidad Católica del Perú, Lima. 2008 The astronomical significance of ritual movements in the calendar of Cuzco. En Pre-Columbian Landscapes of Creation and Origin, editado por J. E. Staller, pp. 250-267. Springer, New York.

2009 El Calendario Inca: Tiempo y Espacio en la Organización Ritual del Cusco; La Idea del Pasado. Fondo Editorial del Congreso del Perú y Pontificia Universidad Católica del Perú, Lima, en prensa. 


\section{Notas}

1 El gnomon, es una columna vertical enterrada parcialmente en el suelo que sirve para calcular el tiempo mirando el Sol gracias a la sombra que la columna proyecta sobre una superficie plana con marcas. La etnografía señala que era de uso común entre los atacameños (Grebe e Hidalgo 1988:77).

2 Medida angular medida desde el norte geográfico o verdadero en dirección este.

3 Del conjunto cerámico recuperado en Viña del Cerro destaca el tipo local Punta Brava con $71,2 \%$, que correspondería a grandes vasijas usadas para contener granos, agua y otros líquidos. Cerámica Copiapó con sus variantes Negro sobre Rojo y Crema con un 14,7\%. Cerámica Café Gris Alisado con un $9,2 \%$. Y cerámica Diaguita de aculturación incaica Negro sobre Crema o Negro y Rojo sobre Crema, además de Negro Bruñido, que representan sólo el 3\% del conjunto total con formas de keros, aríbalos y escudillas extendidas (Castillo 1997:237).

4 Datos entregados por el arqueólogo Rubén Stehberg (comunicación personal 2006), dan cuenta de la existencia de un agujero o "pozo" encontrado en las ruinas incaicas de Chada, $55 \mathrm{~km}$ al sur de la ciudad de Santiago. Este podría no sólo haber servido como lugar para depositar algún tipo de ofrenda y/o almacenar agua en la cumbre del pukara, sino también ser considerado un ushnu.

5 Es el punto en el cual la vertical del lugar (perpendicular al plano fundamental o plano del horizonte) intercepta a la esfera celeste por encima de la cabeza del observador.

6 O nadir, corresponde al punto diametralmente opuesto al cenit, es decir, es la prolongación bajo los pies del observador de la vertical del lugar sobre la esfera celeste.

$7 \quad$ Es la trayectoria del Sol en la esfera celeste correspondiente a un año, y tiene una diferencia de ángulo con el ecuador celeste de $23,5^{\circ}$ en la actualidad.
8 Implementación del modelo IGRF-10 de la IAGA (International Association of Geomagnetism and Aeronomy) http://recursos.gabrielortiz.com/calculadora_declinacion/ entrada.asp

9 Bustamante, P. (c) 141.465.

10 Esta aproximación podría variar en $+/-1^{\circ}$, debido a la distorsión que pudiera ocasionar el lente de la cámara o la forma en la que se tomó la fotografía desde el ushnu.

11 Salida Sol solsticio diciembre (SSSD), Puesta Sol solsticio diciembre (PSSD), Salida Sol solsticio junio (SSSJ), Puesta Sol solsticio junio (PSSJ), Salida Sol equinoccio (SSEQ), Puesta Sol equinoccio (PSEQ), Salida Luna extrema norte (SLEN), Puesta Luna extrema norte (PLEN), Salida Luna extrema sur (SLES), Puesta Luna extrema sur (PLES), Salida Alfa Centauro (SAC), Puesta Alfa Centauro (PAC).

12 El cerro Manchado visto desde Viña del Cerro tiene un acimut de $100^{\circ}$ aproximadamente. De acuerdo al análisis de la carta solar para Copiapó (Stuven 1972) y el conocimiento de la curva que realiza el Sol desde el horizonte teórico o matemático, podríamos sugerir que el Sol visto desde la plaza debiera salir por este hito topográfico entre finales de octubre y comienzos de noviembre, fechas que coinciden con los días del pase del Sol por el cenit en la ciudad del Cusco. Esta observación podría tener cierta relevancia si consideramos que en Mesoamérica se manejaba un calendario fijo de días que tomaba como punto de referencia los pasos del Sol en el cenit en la latitud $15^{\circ}$ (Broda 2004 [2003]). Y también el reciente trabajo de Zuidema (2009), quien asegura que los incas conocían la extensión del año (días fijos) gracias a observaciones lunares, solares y estelares.

13 Dejamos abierta la posibilidad a que nuevos trabajos puedan falsear esta hipótesis, considerando p.ej. la existencia de un número distinto de huayras en el lugar. 